\title{
Electrochemical investigation of biomolecular interactions between platinum derivatives and DNA by carbon nanotubes modified sensors
}

\author{
Ece Yapasan ${ }^{\mathrm{a}, \mathrm{b}}$, Ayfer Caliskan ${ }^{\mathrm{b}}$, Hakan Karadeniz ${ }^{\mathrm{b}}$, Arzum Erdem ${ }^{\mathrm{b}, *}$ \\ a Izmir Institute of Technology, Faculty of Science, Chemistry Department, 35430 Urla, Izmir, Turkey \\ ${ }^{\mathrm{b}}$ Ege University, Faculty of Pharmacy, Analytical Chemistry Department, 35100 Bornova, Izmir, Turkey
}

\section{A R T I C L E I N F O}

\section{Article history:}

Received 2 June 2009

Received in revised form 12 October 2009

Accepted 16 October 2009

\section{Keywords:}

Carbon nanotubes

DNA interactions

Electrochemical impedance spectroscopy

Differential pulse voltammetry

cis-Diamminedichloroplatinum(II)

Oxaliplatin

\begin{abstract}
A B S T R A C T
The biomolecular interactions of platinum derivatives widely used as anticancer drugs: cisdiamminedichloroplatinum(II) and oxaliplatin with calf thymus double-stranded DNA were studied using differential pulse voltammetry (DPV) and electrochemical impedance spectroscopy (EIS) in combination with single-walled carbon nanotubes modified graphite electrode (SWCNTs-GE) and unmodified graphite electrode (bare GE). The performance of these biomolecular interactions were explored at the electrode surface by monitoring the changes at guanine oxidation signal in terms of optimum interaction times by comparing the results of SWCNTs-GE with bare one. The features of these electrochemical sensors based on carbon nanotubes for monitoring of biomolecular interactions were discussed and compared with the earlier conventional ones.
\end{abstract}

(c) 2009 Elsevier B.V. All rights reserved.

\section{Introduction}

During early sixties, many electrochemical approaches have been developed for analyzing or quantification of nucleic acids and its interactions [1-13]. Various types of affinity biosensors as "Genosensor" for nucleic acid recognition processes have been rapidly developed incorporating different nanomaterials for the detection of specific nucleic acid sequences related with genetic and infectious diseases. Immediate applications will include direct quantification of DNA samples for use in sequencing or polymerase chain reactions, or pharmaceutical testing and quality control. Detection of biomolecular interaction of some anticancer drugs (Epirubicin, Mitoxantrone, Echinomycin, Daunomycin, etc.), several kinds of carcinogens (Benzo[a]pyrene, etc.) and toxins (Microcystin, etc.) with DNA is the major branch of electrochemical biosensors. Variety of techniques have been used to detect interaction of cancer drugs with DNA [3-5].

Nanotechnology has a key importance for the development of challenging tools, including biosensor technologies. Carbon nanotubes were discovered in 1991 by Lijima [14]. They are large macromolecules that are unique for their size, shape, and remarkable physical properties. Their intriguing structures have sparked

\footnotetext{
* Corresponding author. Tel.: +90 232343 4000x5131; fax: +90 2323885258 .

E-mail addresses: arzum.erdem@ege.edu.tr, arzume@hotmail.com (A. Erdem).
}

much excitement in the recent years and a large amount of research has been dedicated to their understanding. The nanotubes have a very broad range of electronic, thermal, and structural properties that change depending on the different kinds of nanotube. To make things more interesting, besides having a single cylindrical wall (SWNTs), the nanotubes can have multiple walls (MWNTs) cylinders inside the other cylinders.

The investigations at chemistry side based on DNA-drug interactions may provide new compounds, that could be tested for an effect on a biochemical target, and also used as promising hybridization indicator in combination with DNA hybridization biosensors based on nanomaterials, which will further become DNA microchip systems $[6,12,13,15]$.

Recent advancements in the development of biosensors with the advances in nanotechnology provide novel tools to develop new techniques for monitoring biomolecular recognition events at the solid surfaces, or in the solution phase. Typical applications include environmental monitoring and control, DNA diagnosis, and chemical measurements in the agriculture, food and drug industries $[6,12,13,15-24]$. Electrochemical detection of nucleic acids related to the specific gene for the breast cancer BRCA1 mutation provided the enhanced signals contrast to bare one by using MWNT modified glassy carbon electrode [25]. A sensitive and low cost detection of specific DNA hybridization at the surface of MWCNT modified disposable graphite electrode was performed employing a simple electrochemical protocol by Erdem et al. [6]. In another work of our group, the application of MWCNT-screen printed electrodes was 


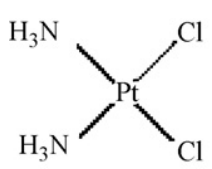

(a)

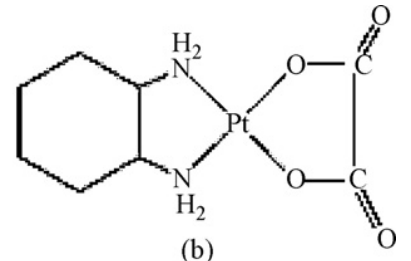

(b)

$\mathrm{O}$
Fig. 1. Chemical structures of (a) cisplatin (cis-DDP) and (b) oxaliplatin (OXP).

successfully presented for monitoring of DNA hybridization based on specific sequences on Hepatitis B virus DNA [21].

In our recent study [15], the disposable graphite electrodes were modified by using the single-walled carboxylic acid functionalized carbon nanotubes and their surface morphologies were then explored using scanning electron microscopy (SEM) analysis. These modified electrodes were used for electrochemical detection of nucleic acids and the interaction between an anticancer drug, Daunorubicin and double-stranded DNA based on the higher signal enhancement contrast to bare electrodes.

The aim of this study was to explore the application of carboxylic acid functionalized single-walled carbon nanotubes modified graphite sensors (SWCNTs-GE) for electrochemical monitoring of biomolecular interactions of platinum derivatives widely used as anticancer drugs: cis-diamminedichloroplatinum(II) (cisDDP) and oxaliplatin (OXP) with calf thymus double-stranded DNA were studied by using differential pulse voltammetry (DPV) and electrochemical impedance spectroscopy (EIS).

In current situation, cis-DDP is widely used in chemotherapy of many types of cancer (Fig. 1). Oxaliplatin is a platinum-based chemotherapy drug which belongs to the same family as cisplatin [26]. Besides effectiveness of drugs, it gives many side effects which limit the clinical application of this compound. For this reason, studies are focused on designing a novel analog of cis-DDP that is equally effective in chemotherapy, but less toxic [26].

The performance characteristics of these biomolecular interactions were explored at the electrode surface by measuring the changes at guanine signal in terms of optimum interaction times by comparing the results of SWCNTs-GE with bare one. The features of these electrochemical sensors based on carbon nanotubes for monitoring of biomolecular interactions were discussed and compared with the earlier conventional ones.

\section{Experimental}

\subsection{Apparatus and chemicals}

All experimental measurements were carried by using AUTOLAB - PGSTAT 302 electrochemical analysis system supplied with a FRA 2.0 module for impedance measurements, and GPES 4.9 software package (Eco Chemie, The Netherlands). For electrochemical measurements, the differential pulse voltammetry (DPV) and electrochemical impedance spectroscopy (EIS) were used. The three-electrode system was consisted of the graphite electrode (GE), an $\mathrm{Ag} / \mathrm{AgCl} / \mathrm{KCl}$ reference electrode (BAS, Model RE-5B, W. Lafayette, USA) and a platinum wire as the auxiliary electrode. The EIS measurements were performed in the Faraday cage (Eco Chemie, The Netherlands).

Platinum complexes and calf thymus double-strand DNA were purchased from Sigma (Germany). Single-walled carboxylic acid (80-90\%) functionalized carbon nanotubes (diameter $4-5 \mathrm{~nm}$; length 500-1500 $\mathrm{nm}$ bundles) was purchased from Aldrich.

DNA stock solutions $(1000 \mathrm{mg} / \mathrm{L})$ were prepared with $10 \mathrm{mM}$ Tris- $\mathrm{HCl}$ containing $1 \mathrm{mM}$ EDTA (TE; pH 8.00) and kept frozen. cisDDP and OXP stock solutions were prepared in dimethyl sulfoxide
(DMSO). DNA and drugs were further diluted using $0.5 \mathrm{M}$ acetate buffer containing $20 \mathrm{mM} \mathrm{NaCl}$ (ABS; pH 4.80).

Other chemicals were in analytical reagent grade and they were supplied from Sigma and Merck. All buffer solutions were prepared using deionized and autoclaved water.

\subsection{Procedure}

The immobilization of nucleic acids/detection of biomolecular detection cycle was performed at freshly SWCNTs modified disposable graphite electrodes (SWCNTs-GEs) and also bare ones. All experiments were carried out at room temperature $\left(25^{\circ} \mathrm{C}\right)$.

The preparation of SWCNTs solution, and SWCNTs modified graphite electrodes, and the $50 \mu \mathrm{g} / \mathrm{mL}$ DNA modified SWCNTs-GE were done according to the information given in earlier report $[6,15]$. Easy surface modification of disposable graphite electrodes with SWCNT was performed properly via passive adsorption, and DNA immobilization was, respectively, done by the formation of covalent coupling between the carboxylated ends of nanotubes and the amine group in the guanine bases of nucleic acid without using any covalent agents.

\subsection{Interaction of cis-DDP with DNA}

DNA modified electrodes were immersed into the vials containing $5 \mu \mathrm{g} / \mathrm{mL}$ cis-DDP during a required interaction according to wet-adsorption process [27]. Each electrode was then rinsed with ABS for $10 \mathrm{~s}$. The voltammetric transduction was then performed.

\subsection{Interaction of OXP with DNA}

DNA modified electrodes were immersed into the vials containing $5 \mu \mathrm{g} / \mathrm{mL}$ OXP during a required interaction according to wet-adsorption process [27]. Each electrode was then rinsed with $\mathrm{ABS}$ for $10 \mathrm{~s}$. The voltammetric transduction was then performed.

\subsection{Voltammetric transduction}

DPV measurements were performed for detection of biomolecular interactions in ABS by scanning between $+0.20 \mathrm{~V}$ and $+1.30 \mathrm{~V}$ potential range at the pulse amplitude: $50 \mathrm{mV}$ and the scan rate: $30 \mathrm{mV} / \mathrm{s}$.

Repetitive measurements were carried out by freshly prepared CNT modified disposable GEs and bare ones by repeating the above assay formats in different concentration levels of drugs and interaction times.

\subsection{Impedance measurements}

After the surface modification of the electrodes by SWCNTs and then dsDNA, the EIS measurement was done accordingly to the procedure given below. In order to present the effect of interaction of cis-DDP, or OXP with dsDNA at the surface of SWCNTs modified GE, the EIS measurement was also performed in the same conditions.

The EIS measurements were performed by using $2.5 \mathrm{mM}$ $\mathrm{K}_{3}\left[\mathrm{Fe}(\mathrm{CN})_{6}\right] / \mathrm{K}_{4}\left[\mathrm{Fe}(\mathrm{CN})_{6}\right](1: 1)$ mixture as a redox probe prepared in $0.1 \mathrm{M} \mathrm{KCl}$. The impedance was measured in the frequency range from $100 \mathrm{mHz}$ to $100 \mathrm{KHz}$ in a potential of $0.23 \mathrm{~V}$ versus $\mathrm{Ag} / \mathrm{AgCl}$ (sat. $\mathrm{KCl}$ ), with a voltage amplitude of $10 \mathrm{mV}$. The frequency interval divided into 51 logarithmically equidistant measure points. The respective semicircle diameter corresponds to the charge-transfer resistance, $R_{\mathrm{ct}}$, the values of which are calculated using the fitting programme AUTOLAB 302 (FRA, version 4.9 Eco Chemie, The Netherlands). 

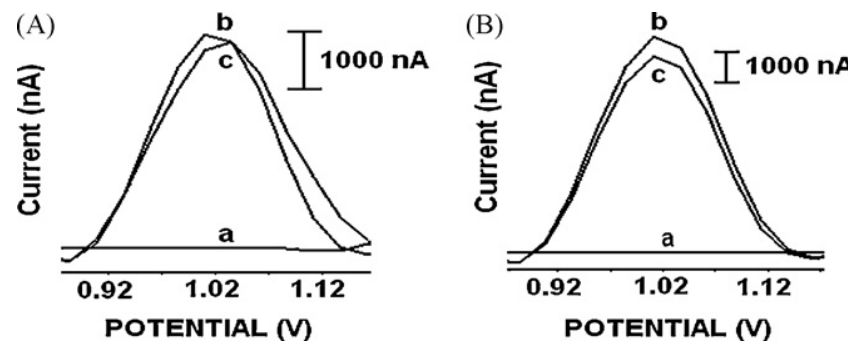

Fig. 2. Voltammograms showing guanine oxidation signals in the absence/presence of biomolecular interactions between cis-DDP and DNA: (A) bare electrodes, (B) SWCNTs modified electrodes, (a) cis-DDP signal in the absence of DNA, (b) guanine signal before interaction with cis-DDP, and (c) guanine signal after interaction with cis-DDP.

\section{Results and discussion}

Disposable SWCNTs-GE were aimed to use in our study for electrochemical monitoring of biomolecular interactions between drugs and calf thymus double-stranded DNA by using DPV and EIS in combination with single-wall carbon nanotubes modified graphite electrode (SWCNTs-GE) and unmodified graphite electrode (bare GE). Under this scope, the platinum derivatives drugs: cis-DDP and OXP were used as the target compounds for biomolecular interactions with DNA.

The representative voltammograms were shown in Fig. 2 and the magnitudes of guanine oxidation signals were measured at $+1.00 \mathrm{~V}$ in the absence/presence of cis-DDP interactions with DNA in order to present the electrochemical performance of bare electrodes (Fig. 2A) to the SWCNTs modified ones (Fig. 2B). No significant signal related to cis-DDP was obtained with bare electrode and SWCNTs modified one at the same peak position of guanine.

An enhancement at guanine signal was similarly also observed when nanomaterial based electrode was used in parallel to the results reported in previous reports $[6,15]$. To obtain an enhanced guanine response could be a result of high electrical conductivity of SWCNTs, and electrocatalytic action of SWCNTs able to promote electron transfer reactions.

After interaction of cis-DDP with DNA immobilized onto the surfaces of bare GE and SWCNTs modified ones, there was the decrease ratio, respectively, as $22.5 \%$ and $13.4 \%$ obtained at the oxidation signals of guanine in 30 min interaction time (shown also in Fig. 3). In a shorter interaction time as $5 \mathrm{~min}$, this decrease was gradually smaller (respectively, $4.2 \%$ and 6.5\%) than the recorded ones above.

Similarly, the representative DPVs were recorded by measuring the oxidation signals of guanine in the absence/presence of interaction between OXP and DNA at the surface of bare electrodes (Fig. 4A) to the SWCNTs modified ones (Fig. 4B). No oxidation signal of OXP was observed with bare electrode and SWCNTs modified one in the same measurement scale with electroactive DNA base, guanine.
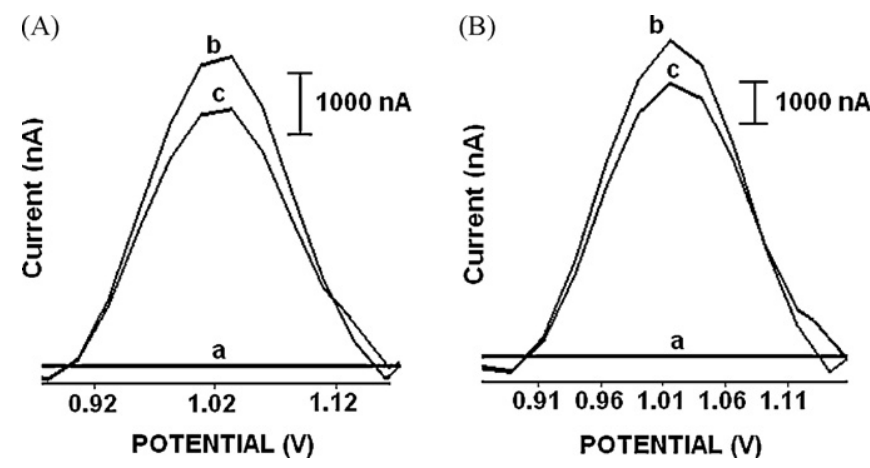

Fig. 4. Voltammograms showing guanine oxidation signals in the absence/presence of biomolecular interactions between OXP and DNA: (A) bare electrodes, (B) SWCNTs modified electrodes, (a) OXP signal in the absence of DNA, (b) guanine signal before interaction with OXP, and (c) guanine signal after interaction with OXP.

After interaction of OXP with DNA immobilized onto the surfaces of bare GE and SWCNTs modified ones, there was the decrease ratio, respectively, as $22.0 \%$ and $18.6 \%$ obtained at guanine signals in 30 min interaction time (shown also in Fig. 5).

The observed decrease in the guanine signal can be attributed to the binding of these platinum derivatives specifically to guanine bases in the skeleton of DNA. This phenomenon could be explained by the shielding of oxidizable groups of electroactive DNA base, guanine while cis-DDP, or similarly OXP interact with nucleic acids at the electrode surface or in a solution phase, similarly explained in earlier reports [28-31]. One of the previous studies presented the electrochemical detection of carboplatin interaction with DNA by using glassy carbon electrode [28], it was concluded that the platin compounds can bind covalently to DNA with cross links between two bases on opposite strands of DNA helix and intrastrand cross links two bases on the same DNA strand. Brabec also presented the results that show a linear decrease at guanine signal upon increasing concentrations of [Pt-(dien) $\left.\left(\mathrm{H}_{2} \mathrm{O}\right)\right]^{2+}$ with DNA by paraffin-wax impregnated spectroscopic graphite electrode [29].

Accordingly, the reproducible and sensitive detection scheme resulted in a shorter time was presented herein for electrochemical investigation of biomolecular recognitions of the anticancer drug, cis-DDP with DNA and similarly for another anticancer agent OXP by the advantages of SWCNTs modified disposable graphite electrodes.

The voltammetric results for the biomolecular recognition process were also complemented successfully with the ones investigated herein by electrochemical impedance spectroscopy [32]. EIS has been widely used to study a variety of other electrochemical systems, including corrosion, electrodeposition, batteries and fuel cells. However, only recently have impedance methods been applied in the field of biosensors. Given their ability to sense charge transfer resistance $\left(R_{\mathrm{ct}}\right)$ and double layer capacitance $(\mathrm{Cd})$ values,
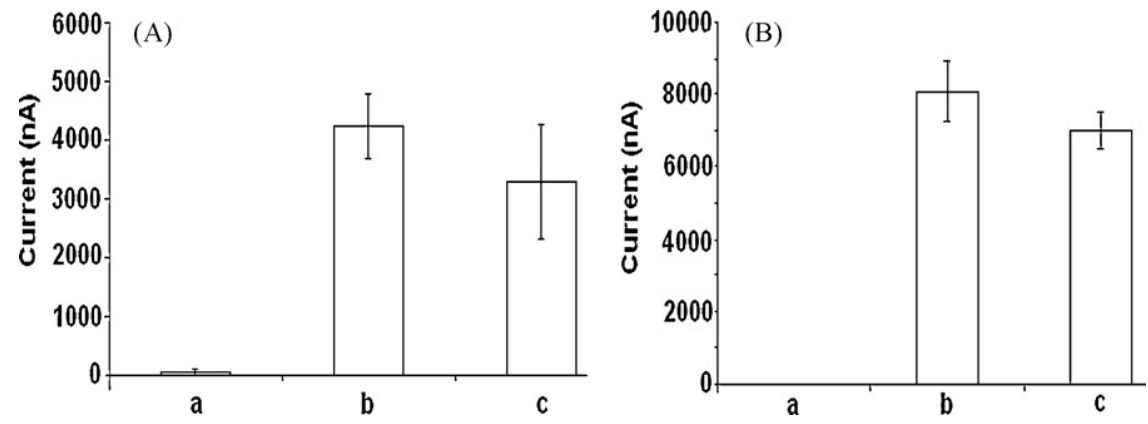

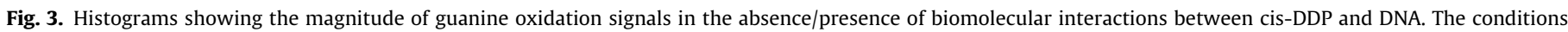
same in Fig. 2. 

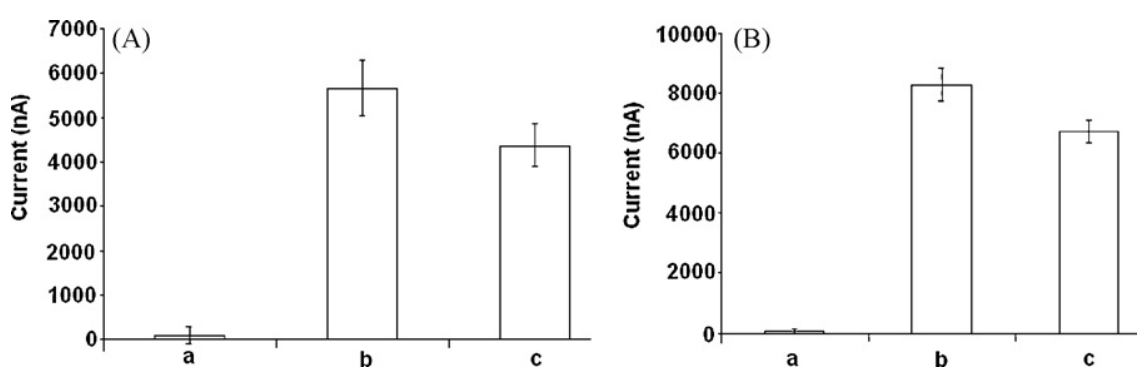

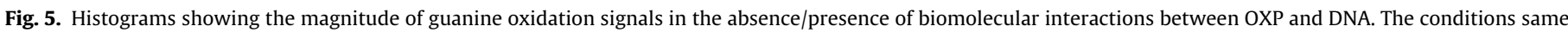
in Fig. 4.

application should be possible for several different types of sensing schemes, with numerous recognition agents [32].

After the modification of the surface of graphite electrode with carbon nanotubes, the average $R_{\mathrm{ct}}$ value (shown in Fig. 6B-a) was calculated as $145 \Omega$, which is three times larger than the one obtained by bare electrode (not shown) in parallel to the results obtained by using bare gold electrode and multiwalled carbon nanotube modified one [33]. The change of the $R_{\mathrm{ct}}$ value after modification was a strong proof that CNT had been immobilized onto the electrode surface. The negatively charged phosphate backbone of double-stranded DNA prevented redox couple, $\left[\mathrm{Fe}(\mathrm{CN})_{6}\right]^{3-/ 4-}$ from reaching the electrode surface, leading to almost three folds larger $R_{\mathrm{ct}}$ value (shown in Fig. 6B-b). These results clearly presented that the modification of carbon nanotubes onto the graphite surface improved the immobilization capacity of dsDNA similarly to the earlier reports using nanomaterials $[15,34]$. Correspondingly to the surface confined biomolecular interaction with cis-DDP, an increase level was found $32.4 \%$ at the $R_{\mathrm{ct}}$ value, that may possibly
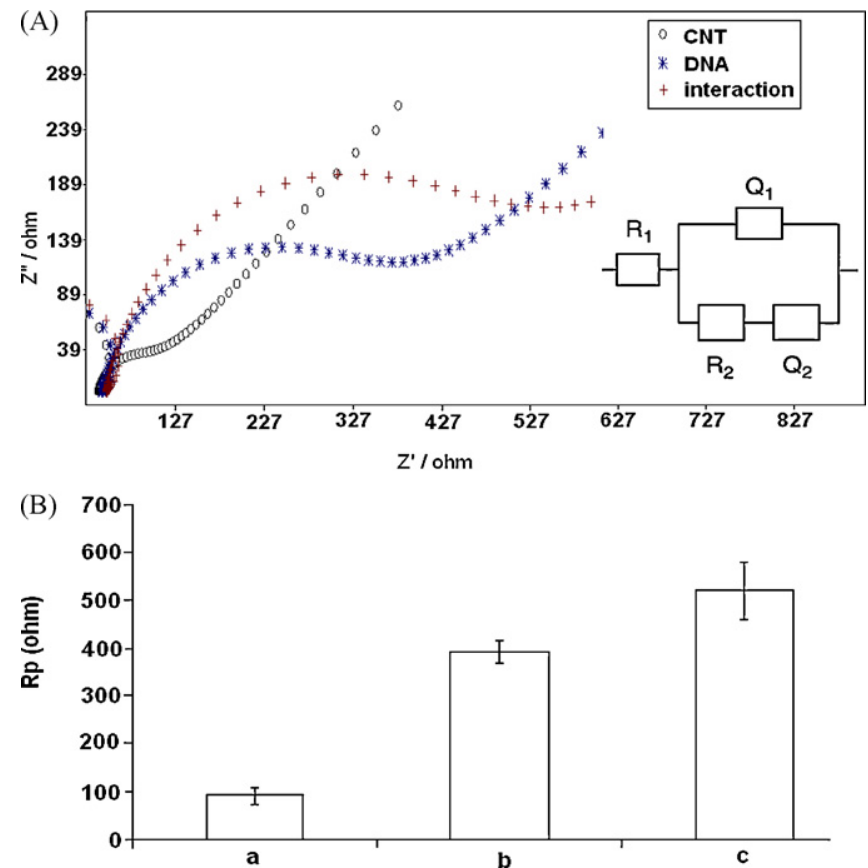

Fig. 6. (A) Nyquist diagrams recorded at SWCNTs modified electrode after interaction of cis-DDP with DNA. Inset was the equivalent circuit model used to fit the impedance data, the parameters of which are listed in the text; R1 is the solution resistance; the constant phase element $\mathrm{Q} 1$ is then related to the space charge capacitance at the DNA/electrolyte interface; R2 is related to the charge transfer resistance at the DNA/electrolyte interface; the constant phase element Q2 is the Warburg impedance due to mass transfer to the electrode surface. (B) Histograms representing the $R_{\mathrm{ct}}$ values measured after electrode surface modification, respectively, with (a) SWCNTs and (b) dsDNA. After the interaction of cis-DDP with dsDNA at the surface of SWCNTs-GE the $R_{\mathrm{ct}}$ was also recorded and represented as (c).
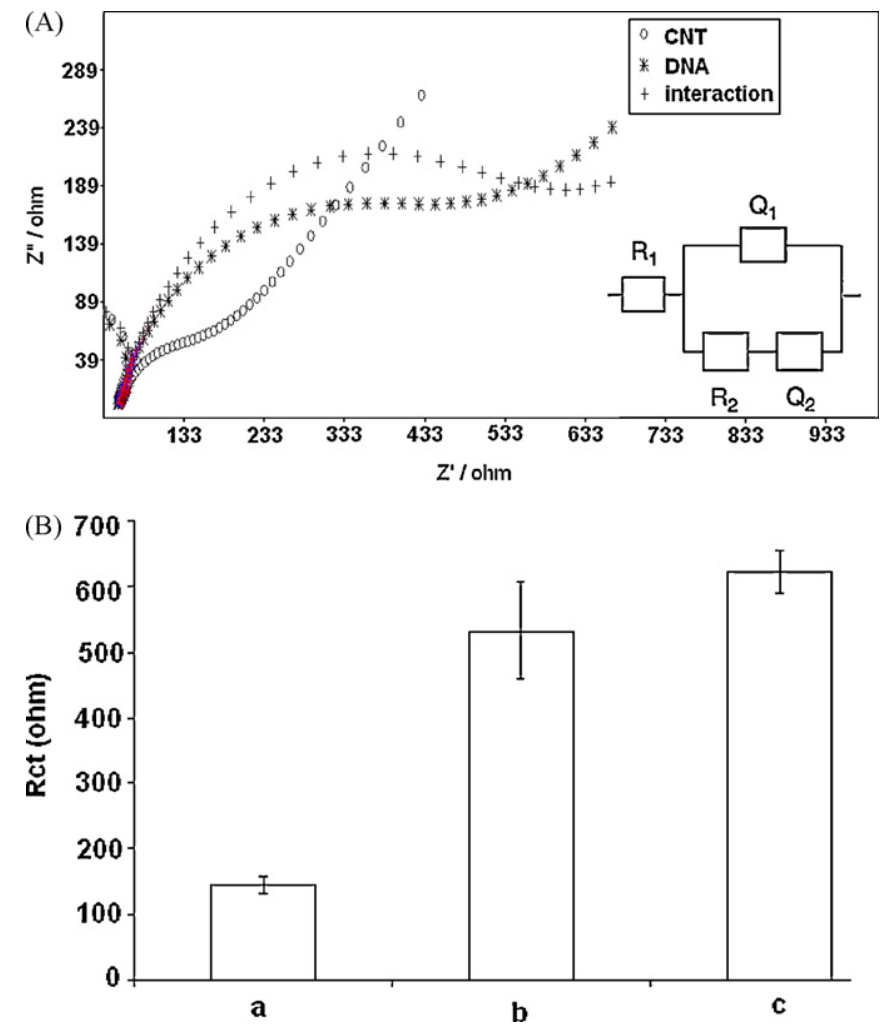

Fig. 7. (A) Nyquist diagrams recorded at SWCNTs modified electrode after interaction of OXP with DNA. (B) Histograms representing the $R_{\mathrm{ct}}$ values measured after electrode surface modification, respectively, with (a) SWCNTs and (b) dsDNA. After the interaction of OXP with dsDNA at the surface of SWCNTs-GE the $R_{\mathrm{ct}}$ was also recorded and represented as (c).

indicate that there was a specific binding of platinum complexes to the guanine bases of in the double helix form of DNA.

The $R_{\mathrm{ct}}$ values in the absence/presence of interaction between OXP and dsDNA were recorded, and similar increase at the $R_{\mathrm{ct}}$ values (shown in Fig. 7B) was also of interaction of OXP obtained in the presence with DNA.

\section{Conclusion}

In this study, the reproducible and sensitive detection scheme resulted in a shorter time was presented for electrochemical investigation of biomolecular recognitions of the anticancer drug, cis-DDP with DNA and similarly for another anticancer agent OXP by the advantages of SWCNTs modified disposable graphite electrodes resulting with an enhancement at the guanine signal. There have not yet been any reports in the literature for electrochemical investigation of interaction between platinum derivatives and nucleic acids using carbon nanotubes modified electrodes. Voltam- 
metric results were consistent with the ones of electrochemical impedance spectroscopy. On the basis of DPV and EIS results, it can be concluded that DNA could be immobilized properly onto the surface of SWCNTs modified electrodes.

These studies have a great importance for further development of nanomaterial based electrochemical sensors, that may also be used for monitoring of different biomolecular recognitions, such as protein-DNA interactions, or toxin effects for environmental monitoring.

\section{Acknowledgements}

Authors acknowledge the financial support from Turkish Scientific and Technological Research Council (TUBITAK Project No.106S181). A.E. would like to express her gratitude to the Turkish Academy of Sciences (TUBA) as the associate member of TUBA for their support. E.Y. and A.C. acknowledge for the master project scholarship obtained from TUBITAK.

\section{References}

[1] E. Palecek, Nature 188 (1960) 656-657.

[2] M.I. Pividori, A. Merkoci, S. Alegret, Biosens. Bioelectron. 15 (2000) 291-303.

[3] J. Wang, Nucleic Acids Res. 28 (2000) 3011-3016.

[4] E. Palecek, M. Fojta, Anal. Chem. 73 (2001) 75A-83A

[5] A. Erdem, M. Ozsoz, Electroanalysis 14 (2002) 965-974.

[6] A. Erdem, P. Papakonstantinou, H. Murphy, Anal. Chem. 78 (2006) 6656-6659.

[7] J. Wang, A.N. Kawde, A. Erdem, M. Salazar, Analyst 126 (2001) 2020-2024.

[8] A. Erdem, M.I. Pividori, A. Lermo, A. Bonanni, M. del Valle, S. Alegret, Sens. Actuators B: Chem. 114 (2006) 591-598.

[9] J. Wang, D. Xu, A. Erdem, R. Polsky, M. Salazar, Talanta 56 (2002) 931-938.
[10] J. Wang, G.U. Flechsig, A. Erdem, O. Korbut, P. Gründler, Electroanalysis 16 (2004) 928-931.

[11] A. Erdem, D. Ozkan Ariksoysal, H. Karadeniz, P. Kara, A. Sengonul, A.A. Sayiner, M. Ozsoz, Electrochem. Commun. 7 (2005) 815-820.

[12] A. Erdem, F. Sayar, H. Karadeniz, G. Guven, M. Ozsoz, E. Piskin, Electroanalysis 19 (2007) 798-804.

[13] H. Karadeniz, A. Erdem, A. Caliskan, C.M. Pereira, E.M. Pereira, J.A. Ribeiro, Electrochem. Commun. 9 (2007) 2167-2173.

[14] S. Lijima, Nature 354 (1991) 56-58.

[15] A. Erdem, H. Karadeniz, A. Caliskan, Electroanalysis 21 (2009) 464-471.

[16] W.G. Kuhr, Nat. Biotechnol. 18 (2000) 1042-1043.

[17] J. Wang, Analyst 130 (2005) 421-426.

[18] J. Wang, Anal. Chim. Acta 469 (2002) 63-71.

[19] A. Erdem, Talanta 74 (2007) 318-325.

[20] K. Besteman, J.O. Lee, F.G. Wiertz, H.A. Heering, C. Dekker, Nano Lett. 3 (2003) 727-730.

[21] H. Karadeniz, A. Erdem, A. Caliskan, Electroanalysis 20 (2008) 1932-1938.

[22] A.D. McFarland, R.P. Van Duyne, Nano Lett. 3 (2003) 1057-1062.

[23] F. Patolsky, C.M. Lieber, Mater. Today 8 (2004) 20-28.

[24] S. Mathur, A. Erdem, C. Cavelius, S. Barth, J. Altmayer, Sens. Actuators B: Chem. 136 (2009) 432-437.

[25] J. Wang, A.N. Kawde, M. Musameh, Analyst 128 (2003) 912-916.

[26] C.X. Zhang, S.J. Lippard, Curr. Opin. Chem. Biol. 7 (2003) 481-489.

[27] A. Erdem, in: D. Barcelo (Ed.), Comprehensive Analytical Chemistry in: S. Alegret, A. Merkoci (Eds.), Electrochemical Sensor Analysis, vol. 49, Elsevier, Amsterdam, 2007, pp. 403-411 (Chapter 19).

[28] A.M. Oliveria Brett, H.P. Serrano, A. Macedo, D. Raimundo, M.H. Marques, M.A. La-Scalea, Electroanalysis 8 (1996) 992-995.

[29] V. Brabec, Electrochim. Acta 45 (2000) 2929-2932.

[30] A. Erdem, B. Kosmider, R. Osiecka, E. Zyner, J. Ochocki, M. Ozsoz, J. Pharm. Biomed. Anal. 38 (2005) 645-652.

[31] M. Ravera, G. Bagni, M. Macsini, J.C. Dabrowiak, D. Osella, J. Inorg. Biochem. 101 (2007) 1023-1027.

[32] C. Jiang, T. Yang, K. Jiao, H. Gao, Electrochim. Acta 53 (2008) 2917-2924.

[33] M. Guo, J. Chen, L. Nie, S. Yao, Electrochim. Acta 49 (2004) 2637-2643.

[34] A. Li, F. Yang, Y. Ma, X. Yang, Biosens. Bioelectron. 22 (2007) 1716-1722. 